Artículo de investigación E15A03. Proyecto 25-B8-177 “Prospectiva del transporte de personas y mercancías: el caso de Uber como medio multi-carga”. * Centro de Investigación y Capacitación en Administración Pública (CICAP), Universidad de Costa Rica (UCR). * Recibido: 06.02.2019. * Aprobado versión final: 08.06.2019. JJEL: F02, F10, H11, H83. * Pp. 197-217 doi:10.33571/teuken.v11n17a11

\title{
Evolución del transporte de personas y mercancías. ¿Uber como medio de carga múltiple?
}

\section{Evolution of the transport of people and goods. Uber as a multiple-loading medium?}

\author{
Esteban O. Mora Martínez \\ COSTA RICA
}

Resumen: Este artículo estudia el caso de la empresa Uber Technologies Inc. y su oferta a nivel internacional, abordando cómo su incursión transforma la forma de transporte de personas y mercancías, a pesar de las brechas en las regulaciones nacionales (caso de Costa Rica) e internacionales. La investigación es exploratoria, descriptiva, transversal y cualitativa, con enfoque en el comercio transfronterizo de servicios y en la economía del transporte, provocada por este tipo de plataformas tecnológicas. El resultado es una propuesta de tres escenarios prospectivos, dos de tendencia (servicio público innovador y transporte privado colaborativo) y uno de ruptura (transporte multicarga) para vislumbrar la posibilidad de optimizar el modelo de negocio, a través de catorce variables identificadas y determinadas por cuatro factores: tiempo, costo, social y ambiental.

Palabras clave: Comercio internacional; economía del transporte; transporte; sector terciario; tecnología.

Abstract: This article examines the case of Uber Technologies Inc. and its international offering, addressing how its incursion transforms the way it provides the transportation of people and goods, despite gaps in national (Costa Rican case) and international regulations. The research is exploratory, descriptive, cross-cutting and qualitative, with a focus on cross-border trade in services and the transport economy, caused by such technological platforms. The result is a proposal of three prospective scenarios, two trend scenarios (innovative public service and collaborative private transport) and one rupture (multiload transport) to glimpse the possibility of optimizing the business model, through fourteen variables identified and determined by four factors: time, cost, social and environmental.

Keywords: International trade; transport economy; transport; tertiary sector; technology.

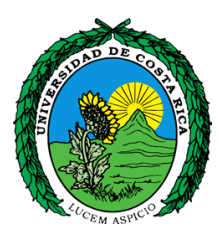

Esteban O. Mora es Doctor en Ciencias de la Administración de la Universidad Estatal a Distancia, Máster en Administración Pública con énfasis en Administración Aduanera y Comercio Internacional y Licenciado en Administración Pública de la Universidad de Costa Rica. Es Profesor del Programa de Posgrado en Administración Pública y de la Escuela de Administración Pública; Investigador y Coordinador del Programa de Investigación e Innovación del Centro de Investigación y Capacitación en Administración Pública, Grupo de Investigación del Centro de Investigación y Capacitación en Administración Pública (CICAP) de la Universidad de Costa Rica.

Contacto: esteban.moramartinez@ucr.ac.cr 


\title{
Evolução do transporte de pessoas e bens. Uber como carga múltipla?
}

\begin{abstract}
Resumo: Este artigo analisa o caso da Uber Technologies Inc. e da sua oferta internacional, abordando a forma como a sua incursão transforma a forma como fornece o transporte de pessoas e bens, apesar das lacunas nos regulamentos nacionais (caso costa-riquenho) e internacionais. A investigação é exploratória, descritiva, transversal e qualitativa, com foco no comércio transfronteiriço de serviços e na economia de transportes, causada por tais plataformas tecnológicas. O resultado é uma proposta de três cenários prospetivos, dois cenários de tendência (serviço público inovador e transporte privado colaborativo) e uma rutura (transporte multicarrego) para vislumbrar a possibilidade de otimizar o modelo de negócio, através de catorze variáveis identificadas e determinadas por quatro fatores: tempo, custo, social e ambiental..
\end{abstract}

Palavras-chave: Comércio internacional; economia de transportes; transporte; sector terciário; tecnologia.

\section{Introducción}

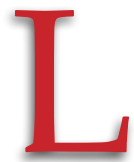

La búsqueda de la eficiencia del transporte no es nueva, así como tampoco lo son sus problemas asociados de contaminación, congestionamiento, transnacionalización, entre otros, que aquejan a todas las sociedades del mundo en diferentes medidas. Si bien el presente artículo no estudia esta situación, sí identifica dentro de ese panorama la incorporación de nuevas tecnologías que permiten a los usuarios un mejor uso y aprovechamiento de los medios de transporte, particularmente, poniendo atención a la utilización de las aplicaciones móviles (apps) para el transporte colaborativo de personas.

Por ejemplo, el transporte privado de personas en Costa Rica ha sido afectado por la transnacionalización, particularmente, con la incursión de la empresa Uber Technologies Inc. (en adelante Uber), pues su figura de plataforma tecnológica para la movilización colaborativa de personas no está tipificada en el marco legal vigente, mientras que los servicios de taxi y de porteo cuentan con leyes específicas que, incluso, cuentan con sanciones para quienes brindan el servicio de traslado de personas sin autorización.

En la actualidad, la empresa Uber, de capital de EEUU, ofrece servicios de transporte privado de personas, con lo cual vino a irrumpir en el status quo de este servicio. Desde el análisis prospectivo que se realiza en las ciencias de la administración, esta situación de competencia puede ser analizada en el marco del Acuerdo General del Comercio de Servicios (AGCS) de la Organización Mundial del Comercio (OMC) en el cual se prevén cuatro modos: 
- Comercio transfronterizo: del territorio de un miembro al territorio de cualquier otro miembro;

- Consumo en el extranjero: en el territorio de un miembro a un consumidor de servicios de cualquier otro miembro;

- Presencia comercial: por un proveedor de servicios de un miembro mediante la presencia comercial en el territorio de cualquier otro miembro; $y$

- Presencia de personas físicas: por un proveedor de servicios de un miembro mediante la presencia de personas físicas de un miembro en el territorio de cualquier otro miembro.

Más allá de la discusión de si el servicio es brindado en condiciones de legalidad en Costa Rica, dichos modos permiten plantear la pregunta: ¿Podría Uber implementar estrategias de diversificación de servicios como transporte de alimentos, bebidas, productos y otro tipo de mercancías con el fin de optimizar la eficiencia del servicio de transporte de personas como un servicio multicarga ante la expansión de la competencia y servicios similares o sustitutivos?

La disrupción causada por las Tecnologías de la Información y Comunicación (TIC) en diferentes áreas del conocimiento como ingeniería, medicina, administración y transporte han facilitado procesos por medio del Internet de las cosas (IoT por sus siglas en inglés) y también el desarrollo de aplicaciones para teléfonos inteligentes y el equipamiento con mayores capacidades para el diagnóstico de las necesidades humanas. Esta realidad ocasiona transformaciones al interior de las organizaciones y genera escenarios futuros para la adaptación de los bienes y servicios que producen para la sociedad.

Esas innovaciones aplicadas al transporte han permito que hoy se conozca con bastante precisión el momento del día que puede tomarse un autobús o un tren, así como el tiempo estimado de llegada al destino, gracias a sistemas de posicionamiento global que, incluso, ya son accesorios integrados en los vehículos más modernos y a los teléfonos inteligentes. La situación también provoca que las organizaciones y los consumidores se adapten al entorno.

Las diversas modalidades de transporte (aéreo, marítimo, ferroviario y terrestre) también han sufrido innovaciones y las cargas pueden seguirse en tiempo real, lo cual sirve para el control de costos, riesgos y eficiencia energética. Al respecto, en un escenario donde convergen las innovaciones tecnológicas para el mejoramiento de los medios y tiempos de transporte, la reducción de la contaminación ambiental 
y el aprovechamiento colaborativo del vehículo, ¿es posible que las aplicaciones permitan la optimización del transporte de personas y mercancías como medios multicarga?

El proyecto de investigación denominado "Prospectiva del transporte de personas y mercancías: el caso de Uber como medio multi-carga" estudia el caso de esta empresa con el fin de determinar si dentro de su evolución existe el espacio de construcción de escenarios futuros que contribuyan a definir un uso más eficiente de los vehículos para el transporte de personas, con la integración del traslado de mercancías, optimizando el uso del vehículo, sus capacidades y la ruta, de modo que se logre un mejor aprovechamiento y un mejor retorno de la inversión.

Durante la formulación y ejecución del proyecto no se encontraron trabajos similares que permitieran explorar escenarios para la modernización de la economía del transporte o del marco legal del transporte de personas y mercancías. Este trabajo, a diferencia de los aportes de Wallsten, (2015), Rauch \& Schleicher (2015), Mora y Arauz (2016), Laurell \& Sandström (2016) y De Moya, (2014), pretende explorar teóricamente posibles escenarios futuros que modelen la oferta de servicios de compañías en el sector transporte de personas y mercancías.

\section{Metodología}

El caso de Uber, como empresa de servicio de transporte privado de personas y de sus posibles negocios derivados (transporte de mercancías), resulta importante estudiarlo para contribuir al conocimiento de disciplinas como la economía del transporte, la administración pública, la administración aduanera y el comercio internacional, ya que el transporte de personas suele estar regulado por leyes específicas y el transporte de carga tiene implicaciones prácticas para las empresas transportistas locales de mercancías. De ahí que este artículo indague por la adaptación del servicio a una modalidad multicarga, a partir de contextualizar el Acuerdo General del Comercio de Servicios como marco de referencia para el transporte de personas y de mercancías en Costa Rica, conocer la evolución del servicio de Uber y proponer variables y escenarios para pronosticar acciones futuras de diversificación del servicio de transporte hacia una modalidad multicarga.

El estudio es exploratorio, descriptivo, transversal y cualitativo. Recurre al análisis documental y a la confrontación de evidencias y datos provistos por fuentes primarias y secundarias en tres fases: 
- Fase I. Marco legal: Se reconocen los aspectos relacionados con la normativa costarricense e internacional para la habilitación de los servicios de transporte, con énfasis en el servicio de personas y mercancías por carretera.

- Fase II. Transformación de servicios: Se identifican las diferentes etapas de diversificación de los servicios de transporte del caso Uber.

- Fase III. Análisis prospectivo: Se realiza una aproximación teórica a las variables y a los escenarios que pueden predecir, prospectivamente, condiciones de desarrollo del transporte multicarga a partir del servicio de transporte privado de personas y mercancías.

Para comprender esta realidad se realiza una revisión documental sobre el transporte público, transporte privado de personas y transporte de mercancías, ya que "las metodologías de investigación cualitativa son maneras posibles de hacer investigación en ciencias sociales. Una de sus formas es la investigación documental" (Gómez et al, 2015, p. 424).

Por medio de técnicas prospectivas, que permiten el análisis de contenidos y realizar proyecciones sin intervenir con los actores involucrados, se extraen aspectos que buscan predecir el comportamiento de este tipo de empresas, "después de visualizar diversos futuros alternativos y seleccionar el 'futurible' (deseable y factible al mismo tiempo)" (Miklos, 2002 , p. 123). En una aproximación al método de escenarios de Godet et al (2000), se muestra en la figura 1 un esquema lineal de análisis con base en elementos documentales para perfilar los escenarios.

Figura 1. Esquema lineal para perfilar escenarios prospectivos

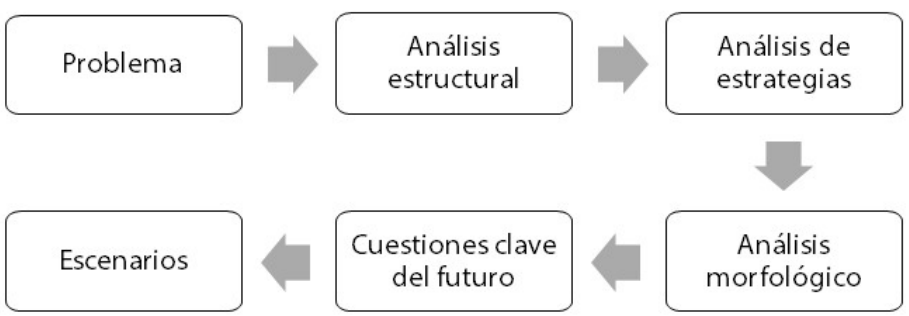

Fuente: Elaboración propia con base en Godet et al. (2000).

El método de escenarios parte de la identificación de un problema, describiendo un conjunto de eventos "que permiten pasar de una situación de origen a una situación futura" (Godet et al, 2000, p. 36). Posteriormente, se aplican tres tipos de análisis:

- Análisis estructural: Permite identificar el sistema y su entorno para determinar variables esenciales. 
- Análisis de estrategia: Caracteriza al actor "en función de sus objetivos, problemas y medios de acción" (Godet et al, 2000, p. 37).

- Análisis morfológico: Utilizado "para descomponer el sistema estudiado en dimensiones esenciales y estudiar las combinaciones posibles de estas diferentes dimensiones, combinaciones que constituyen otras tantas imágenes posibles de futuro" (Godet et al., 2000, p. 37).

Luego, se determinan las cuestiones clave para el futuro, donde se estudia un supuesto en el cual se mantiene una tendencia o, por el contrario, se busca el punto de ruptura de esa tendencia para anticiparse a un cambio probable. Esto lleva a la definición de los escenarios exploratorios (que siguen la tendencia) y de los escenarios normativos (que buscan la alternativa).

En este sentido, el supuesto metodológico que guía la investigación se apoya también en las teorías del comercio de servicios promulgadas por la OMC, estimándose que, a mayor cantidad de asociados de Uber, menor es la rentabilidad para el asociado $y$, por ende, incrementa la oferta de servicios con transporte de mercancías para aumentar la eficiencia en el uso del vehículo, tendiendo al transporte multicarga e impactando en la economía del transporte.

\section{Resultados}

Como se mencionó previamente, no se encontraron trabajos similares que permitieran explorar escenarios para la modernización de la economía del transporte o del marco legal del transporte de personas y mercancías, no obstante, sí se logró evidenciar que el transporte de personas y de mercancías es de vital importancia para las sociedades modernas, e históricamente ha sido una industria sometida a cambios tecnológicos significativos "que han afectado al volumen de movimientos y a la distribución de viajeros y cargas entre las distintas modalidades de transporte" (Rus et al, 2003, p. 1).

La economía del transporte, como teoría de referencia, aborda diez puntos fundamentales para su estudioy comprensión: tecnología de la producción (infraestructura y servicios); tiempo de los usuarios; características de los servicios; inversión óptima en infraestructura; competencia limitada y necesidad de regulación; efectos de red; externalidades negativas; costes (producto, usuario y sociales); obligaciones de servicio público; e infraestructuras y crecimiento (Rus et al, 2003, p. 3).

Los estudios que indagan sobre la percepción del cliente de la calidad en bienes y servicios también pueden ser aplicables al transporte. Aspectos como equipamiento, fiabilidad, capacidad de respuesta, profesionalidad, 
cortesía, credibilidad y seguridad son aspectos que pueden incluirse como categorías de estudio (Lloréns, 1995, p. 38). Por su parte, los estudios de índole operativo, económico y social con técnicas prospectivas se han limitado a casos de la contribución de la logística y el transporte a los cambios de matrices productivas, como el estudio de Mora y Arauz (2016) donde toma en cuenta los criterios de productividad y de mejoramiento de nivel de vida de la ciudadanía.

Dichos enfoques no incluyen estudios prospectivos del transporte desde la perspectiva de la eficiencia de combinar la movilización de personas con el traslado de mercancías, conocido como transporte multicarga, así como tampoco contextualizan la relación con el AGCS de la OMC para la transnacionalización del servicio.

En este sentido, el transporte multicarga como problema de estudio para la comprensión de posibles esquemas de movilización de personas y mercancías, aprovechando vehículos dedicados al transporte colaborativo y privado de personas utilizando la plataforma tecnológica Uber, se examina a continuación por medio de un análisis estructural, que incorpora el marco normativo internacional y nacional de Costa Rica, un análisis de estrategias, que explora la evolución de la empresa Uber, y una análisis morfológico, cuestiones claves del futuro y escenarios, que incluye posibles factores $y$ variables para el servicio privado de personas y mercancías.

\section{Análisis estructural: marco normativo}

Los países miembros de la OMC conscientes de la importancia del comercio de servicios para el crecimiento y el desarrollo de la economía mundial establecieron un marco multilateral de principios y normas denominado Acuerdo General sobre el Comercio de Servicios (AGCS), con el fin de expandir dicho comercio en condiciones de transparencia y de liberalización progresiva, para promover el crecimiento económico de todos los interlocutores comerciales, particularmente, de los países en desarrollo.

En ese contexto, las asimetrías entre países y sus reglamentaciones relacionadas con el suministro de servicios en sus respectivos territorios, es la génesis por la cual surge el AGCS con dos objetivos específicos: facilitar la participación y expandir las exportaciones de servicios, fortaleciendo la capacidad, eficiencia y competitividad nacional en materia de servicios.

Al respecto, Umaña (2016) indica que el sector servicios ha sido el de más rápido crecimiento en la economía mundial en los últimos años, representando esta relación más de dos terceras partes del PIB global, una tercera parte del empleo y el $20 \%$ del comercio internacional. Por ello, se define que el término comprende todo servicio de cualquier sector, excepto los suministrados en ejercicio de facultades gubernamentales, 
es decir, los que no se suministren en condiciones comerciales ni en competencia con uno o varios proveedores. Esto implica aplicar el trato de la nación más favorecida, mediante el cual:

cada Miembro otorgará inmediata e incondicionalmente a los servicios y a los proveedores de servicios de cualquier otro Miembro un trato no menos favorable que el que conceda a los servicios similares y a los proveedores de servicios similares de cualquier otro país (AGCS, Art. II, inciso 1).

Además, surge el concepto de trato nacional, por el cual:

cada Miembro otorgará a los servicios y a los proveedores de servicios de cualquier otro Miembro, con respecto a todas las medidas que afecten al suministro de servicios, un trato no menos favorable que el que dispense a sus propios servicios similares o proveedores de servicios similares (AGCS, Art. XVII, inciso 1).

Los servicios de transporte forman parte de los servicios regulados por el AGCS. Los servicios multimodales son el transporte en el que se utiliza más de un medio de transporte y en las negociaciones del AGCS se trata, fundamentalmente, de los servicios de puerta a puerta, con inclusión del transporte marítimo internacional.

Además, existe el método de la clasificación de los servicios de transporte número MTN.GNS/W/120 del 10 de julio de 1991, que lista la clasificación sectorial de los servicios:

Servicios de transporte por carretera:

a. Transporte de pasajeros.

b. Transporte de carga.

c. Alquiler de vehículos comerciales con conductor.

d. Mantenimiento y reparación de equipo de transporte por carretera.

e. Servicios de apoyo relacionados con los servicios de transporte por carretera.

El AGCS contextualiza el Capítulo 11 del Tratado de Libre Comercio entre República Dominicana, Centro América y EEUU (TLC DR-CAFTA), capítulo que explica el comercio transfronterizo de servicios, mediante el cual se define un marco regulatorio con principios para normar el comercio de servicios entre las partes, a excepción de las medidas adoptadas "en relación con los servicios aéreos, servicios financieros, la contratación pública, los subsidios o donaciones otorgados por el Estado y los servicios o funciones gubernamentales" (COMEX, 2004, p. 20).

Tampoco dispone de medidas para imponer obligaciones sobre el ingreso al mercado de trabajo o del empleo permanente. Incluye la obligación 
de las partes para dar el trato nacional y de nación más favorecida, así como de no adoptar medidas no discriminatorias que restrinjan el acceso al mercado y de no exigir presencia local para el suministro de un servicio, toda vez que las condiciones y limitaciones de su legislación vigente lo establezca. Están incluidas las disposiciones adicionales sobre transparencia, reglamentación nacional, reconocimiento mutuo, transferencias, pagos y compromisos específicos sobre servicios de courier y agentes, distribuidores y representantes de casas extranjeras, protegiéndose los derechos adquiridos de estos últimos.

El TLC prevé, con respecto a los reglamentos nacionales que las partes garantizan, que las leyes que afecten el comercio de servicios deben ser generales y administrarse de manera razonable y con ciertos criterios objetivos, transparentes e imparciales, es decir, que no sean obstáculos al comercio de servicios. También establece la posibilidad de incorporar, previo acuerdo entre las partes, los resultados de negociaciones relacionadas con el AGCS de la OMC, o de negociaciones similares de otros foros internacionales, una vez entradas en vigor.

El transporte público en Costa Rica es una modalidad de servicio público que ha sido autorizado por la Asamblea Legislativa, dada "su importancia para el desarrollo sostenible del país" (Ley No. 7593, Art. 3, inciso a). En este sentido, al ser un país con un Estado social de derecho, cuenta con la Autoridad Reguladora de los Servicios Públicos (Aresep), la cual tiene la misión de asegurar que los servicios públicos regulados se presten en condiciones óptimas de acceso, costo, calidad y variedad para los usuarios. Sin embargo, Uber no está tipificada en la legislación nacional y ha entrado en franca competencia con los servicios de taxi y de porteo.

La Ley No. 3503 indica que el transporte remunerado de personas en vehículos automotores colectivos, excepto los automóviles de servicio de taxi regulado en la Ley 7969, que se lleva a cabo por calles, carreteras y caminos dentro del territorio nacional, es un servicio público regulado, controlado y vigilado por el Ministerio de Obras Públicas y Transportes (MOPT).

También se identifican modificaciones que no necesariamente abarcan el alcance de la normativa estudiada, como por ejemplo, la resolución No. 1353 del 26 de julio de 2018, mediante la cual se crean las Comisiones Mixtas de Transporte Público Urbano, la Comisión Mixta de Transporte Público Interurbana, Comisión Mixta deTransporte Público Interprovincial, la Comisión Mixta de Taxis y la Comisión Mixta de Servicios Especiales, cuyo objetivo fundamental es el diálogo, discusión y propuestas de solución ante los órganos competentes, sobre el cumplimiento efectivo de las políticas públicas vigentes relacionadas con el transporte público, los 
asuntos relacionados con la prestación del servicio público, los sistemas técnicos operacionales, la aplicación tarifaria y los aspectos legales.

Esta disrupción provocada por la tecnología en los servicios de transporte ha tenido diferentes impactos económicos en Costa Rica, similares a los que argumenta Alley (2016) sobre los cambios ocurridos en el transporte en la ciudad de Nueva York relacionados con incrementos en la accesibilidad, productividad en el uso de bienes (vehículos) y movilización de la economía. No obstante, también se presentan externalidades negativas como las que indican Ávalos y Sofía (2015) relacionadas con el sistema de autorregulación de la empresa que incluso busca evadir regulaciones estatales.

El servicio de Uber en Costa Rica está operando en medio de un vacío legal y para llenar ese vacío la Sala Constitucional debe revisar la Ley 8955 para que decida si la eliminación del artículo 323 del Código de Comercio, relacionado con los servicios de transporte de personas tipo "puerta a puerta" (porteo), fue contrario al artículo 11.6 del TLC DR-CAFTA por disminución del acceso al mercado y al artículo 7 de la Constitución Política por oponerse a un tratado público. Mientras esto no ocurra la Ley No. 8955 continuará teniendo plena vigencia y es obligatoria su aplicación, por lo cual, Uber operará en la ilegalidad.

Lo anterior es una nueva condición para el estudio de la economía del transporte, que requiere comprender de mejor manera la economía compartida (por su término en inglés sharing economy) para regularla, así como la interacción entre viajeros, reguladores, empresas y otros actores, como lo han estudiado Rauch \& Schleicher (2015), Davidson \& Fischer (2015), de la Hoz et al (2015), Wallsten (2015) y Chuang et al (2018).

\section{Análisis de estrategias: el caso de Uber}

La idea de Uber nace en 2008 por la necesidad de conseguir un transporte de manera sencilla. En 2009 nace como UberCab, una aplicación para teléfonos inteligentes que permitían conectar una persona con otra (cliente-proveedor) para un servicio de transporte tipo puerta a puerta. En 2010 se convierte en Uber, eliminando la expresión "Cab", utilizada usualmente para los taxis.

En 2011 se internacionaliza en París (Francia), precisamente donde los creadores tuvieron la idea, en los años siguientes continúa su expansión a Australia y Méjico. En 2012 se da la primera diversificación con la entrega de helados a domicilio, es decir, en lugar de solicitar un servicio de transporte puerta a puerta, el cliente solicitaba un servicio a domicilio, lo que se puede identificar como un primer esquema multicarga. Posteriormente, entrega pastelillos como parte de un proceso de responsabilidad social y en 2014 participa con campañas de donación de ropa. 


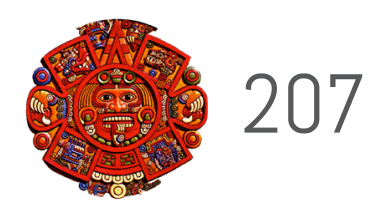

En 2014, lanza UberPool como diversificación del transporte de porteo, esta vez bajo la forma de carpooling (transporte colectivo), donde diferentes usuarios que se dirigen sobre la misma ruta comparten el viaje y los costos. Ese mismo año surge UberMilitary, en la que efectivos militares generaban ganancias al conducir con la plataforma. En 2015 la diversificación avanza, esta vez con UberEats, servicio de comida a domicilio con restaurantes afiliados.

Ese mismo año el servicio es lanzado en Costa Rica, creando polémica y retando al Estado social de derecho y a los servicios de transporte público autorizados: "Uber opera en el país de forma ilegal desde el 21 de agosto de 2015. Ese es hasta el momento el punto de discordia entre las autoridades del Ministerio de Obras Públicas y Transportes (MOPT) y los taxistas" (Granados, 2018).

En septiembre de 2016 la empresa experimenta con vehículos autónomos (sin conductor). En 2017 nace UberFreight, servicio que conecta a las empresas de transporte de carga y a los conductores con los consignantes de manera directa. Dentro de su cultura organizacional utiliza divisiones culturales en procura de entornos de diversidad e inclusión como UberAble, Herencia asiática, UberEqual, UberHue, entre otros.

El análisis de estrategia de Uber se puede sintetizar como una revolución basada en redes digitales para el servicio de transporte con mejoras en dimensiones que van desde el costo hasta la experiencia del usuario (Powers et al, 2016), con la necesidad cada vez mayor de coordinar la formulación de políticas y los incentivos, a fin de cosechar los beneficios potenciales de estos servicios y reducir los efectos negativos (Alemi et al, 2018).

En este sentido, también es posible que la compañía aproveche sus fortalezas de viaje compartido y entrega de alimentos para ramificarse en servicios como la entrega de comestibles que atraen a nuevos usuarios a su plataforma (Singh, 2019). Es decir, existe un esquema de intermediación consolidado; sin embargo, cuando se trata de la distribución de medicamentes, donde conecta a pacientes, farmacias y el servicio de UberEats para despachar medicinas a domicilio, requiere que la plataforma exija que el usuario presente la receta del medicamento que solicita, caso contrario el servicio se limitaría medicamentes de libre venta, ya que el "regente farmacéutico no debe suministrar al paciente ningún medicamento que requiera receta médica" (Mora-Mata, 2019). A pesar de esto, la empresa cuenta con una estructura organizacional que reduce costos y fomenta el trabajo en redes donde combina necesidades de autoempleo, de movilidad por zonas urbanas y de consumo a través de una app que facilita la conexión entre las partes. 
Para Winter (2020) esta es la principal razón por la cual estos servicios son, hasta ahora, utilizados, principalmente, por grupos de usuarios bastante específicos, por ejemplo, para los sistemas de uso compartido de coches en Europa se ha demostrado que estos son utilizados, especialmente, por jóvenes que viven en ciudades y personas con un nivel de educación superior. Independientemente de su estado de ilegalidad o legalidad, el modelo de negocio ofrece aspectos clave del comportamiento del consumidor del siglo XXI que pueden contribuir a mejorar la organización del transporte público de personas y la distribución de mercancías en general.

\section{Análisis morfológico, cuestiones clave del futuro y escenarios}

Los hallazgos de estudios previos de Rauch \& Schleicher (2015), Davidson \& Fischer (2015), de la Hoz et al (2015), Wallsten (2015), Chuang et al (2018), Powers et al (2016), Alemi et al (2018) y Winter (2020) permiten perfilar cuatro factores y catorce variables para explorar los futuros posibles, a partir de la descomposición del sistema y estimar escenarios de la empresa de tecnología según se muestra en la figura 2.

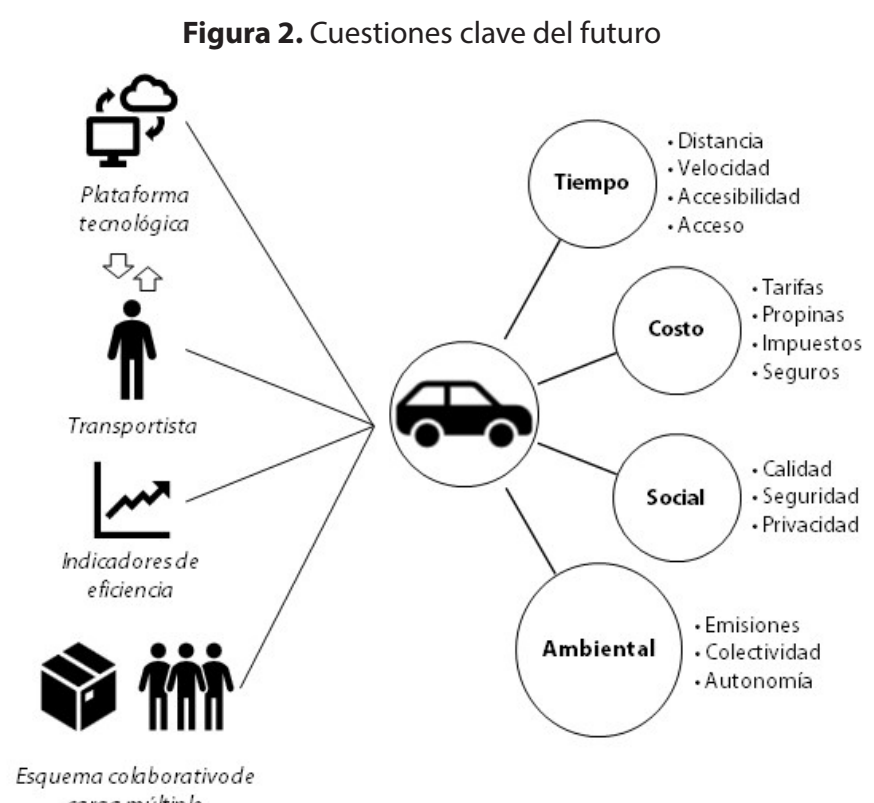

carga múltiple

Fuente: Elaboración propia.

El sistema se descompone en la plataforma tecnológica, el transportista, los indicadores de eficiencia y el esquema colaborativo de carga múltiple o multicarga. Los factores y variables para el servicio privado de personas y mercancías propuestos son: 


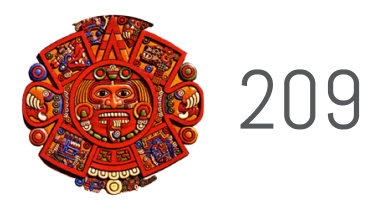

- Tiempo: Está integrado por la distancia de un punto a otro, la velocidad de la provisión del servicio en relación con la ruta óptima trazada al ser un servicio por demanda, la accesibilidad para el pasajero y la carga como condición de versatilidad, y el acceso al servicio por medios digitales.

- Costo: Este factor está asociado con las tarifas del servicio, las posibles propinas por servicios de excelencia, los impuestos al servicio y los seguros para que tanto pasajeros como mercancías estén cubiertos en caso de algún percance o imprevisto.

- Social: Este componente mide la calidad del servicio, la seguridad por parte del proveedor del servicio, tanto de la plataforma electrónica como transportista, y la privacidad con la cual puede realizarse traslado de personas y mercancías.

- Ambiental: Las condiciones relacionadas con un medio equilibrado se estiman en función de la disminución de las emisiones, la eficiencia del transporte basado en la colectividad $y$, al mismo tiempo, la autonomía que brinda solicitar un servicio de transporte sin incurrir en costos propios de combustible, lubricantes, estacionamiento, mantenimiento, entre otros, como gastos asociados a la tenencia de un vehículo.

La aplicación teórica de los factores y variables bajo un esquema lineal permite establecer tres escenarios a partir de sus interacciones: a) servicio público innovador, b) transporte privado colaborativo y c) transporte multicarga.

En la figura 3 se plantea el primer escenario, donde el factor tiempo, integrado por las variables distancia, velocidad, accesibilidad y acceso, junto con el factor social, cuyas variables son calidad, seguridad y privacidad, se estiman como los principales predictores para el escenario de innovación del transporte público, en el cual, el marco regulatorio evoluciona y permite la integración de servicios de porteo y comercio transfronterizo en un mercado de competencia abierta.

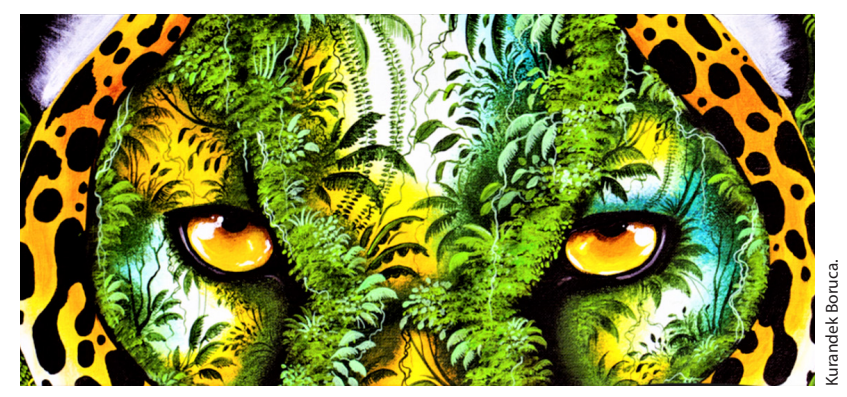


Figura 3. Escenario I. Servicio Público Innovador

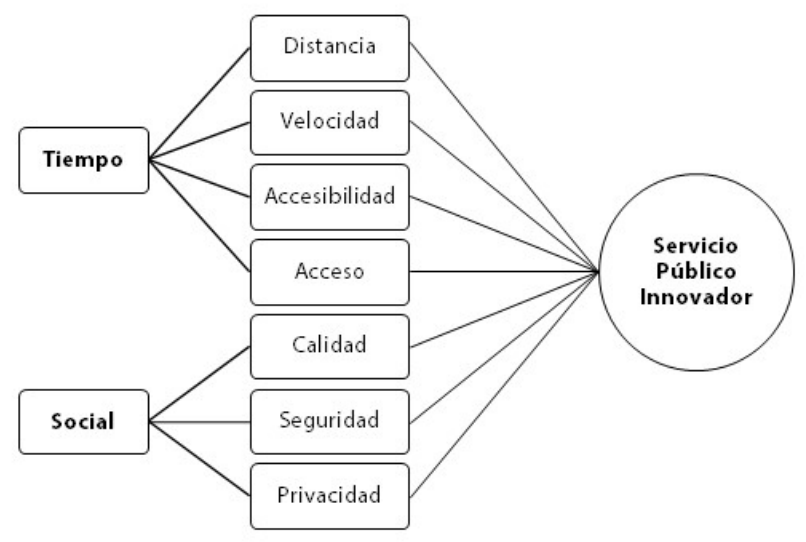

Fuente: Elaboración propia.

El servicio público es innovado por agentes tecnológicos (variable acceso) que facilita que un cliente con una necesidad de llegar a un destino de manera rápida (velocidad de la provisión del servicio), segura y con accesibilidad (versatilidad del vehículo para una persona en cualquier condición física). Además, el servicio se brinda con privacidad y calidad, propiciando un marco regulador actualizado para el servicio público.

Por otra parte, la innovación generada por otros actores del mercado privado de prestadores de servicios de transporte de personas propicia un mercado en competencia con una regulación ampliada a prestadores públicos y privados, mediada por el AGCS y las regulaciones asociadas del TLC DR-CAFTA, de tal forma que los predictores para este escenario son el factor costo (tarifas, propinas, impuestos, seguros) junto con el factor social (calidad, seguridad, privacidad).

El segundo escenario, mostrado en la figura 4, se caracteriza por una transición de los servicios públicos de transporte hacia servicios de porteo, incluidos los servicios de porteo colectivo donde se facilita el conectar a un cliente o varios con un conductor en una ruta específica, generando espacios de colaboración, costos compartidos y disminución del parque automotor en las carreteras, optimizando el servicio de transporte.

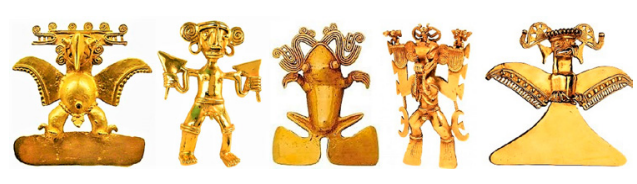


Figura 4. Escenario II. Transporte Privado Colaborativo

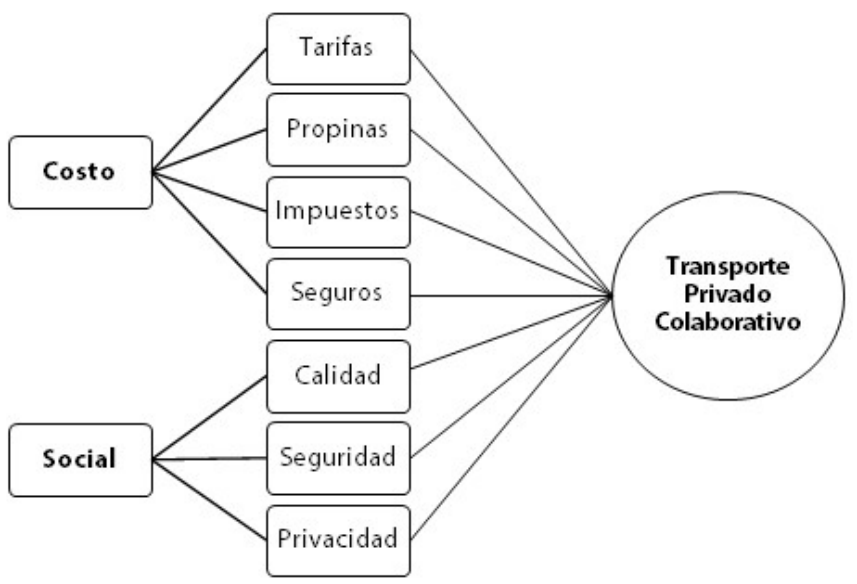

Fuente: Elaboración propia.

Desde el punto de vista teórico, en este escenario la disrupción que realiza Uber en el mercado de transporte público, según Wallsten (2015), está relacionada con un conjunto de quejas sobre el servicio de taxi, que incluyen taxímetros con tarjeta de crédito descompuestas, falta de aire acondicionado o calefacción, mal trato y uso del teléfono celular por parte del conductor.

En dicho contexto, los consumidores encontraron en los servicios de transporte ofrecidos mediante Apps una alternativa a su situación, independientemente de su legalidad y del cumplimiento de requisitos de operación (seguros, licencias, revisiones periódicas del vehículo, seguridad social, impuestos, entre otros).

Otro elemento está relacionado con las redes sociales como catalizadores de la disrupción del mercado en términos de la inserción de la tecnología y de la necesidad de los cambios institucionales. Al respecto, Laurell \& Sandström (2016) exploran el caso de Uber por medio de 6.500 contenidos de redes sociales generados por usuarios de estos servicios y sus hallazgos están relacionados más con la necesidad de un cambio institucional que con una disrupción tecnológica.

En el tercer escenario, sobre el transporte privado de carga múltiple, es decir, de personas y mercancías, es otra innovación posible gracias a la combinación de los cuatro factores y sus correspondientes variables tiempo (distancia, velocidad, accesibilidad, acceso), costo (tarifas, propinas, impuestos, seguros), social (calidad, seguridad, privacidad) y ambiental (emisiones, colectividad, autonomía). En este escenario, mostrado en la figura 5, el factor ambiental es el principal predictor, ya que se estima que 
el usuario tome conciencia sobre su huella de carbono y de la necesidad de optimizar el transporte público y privado, tanto de personas como de mercancías para el aprovechamiento de los recursos naturales.

Figura 5. Escenario III. Transporte Multicarga

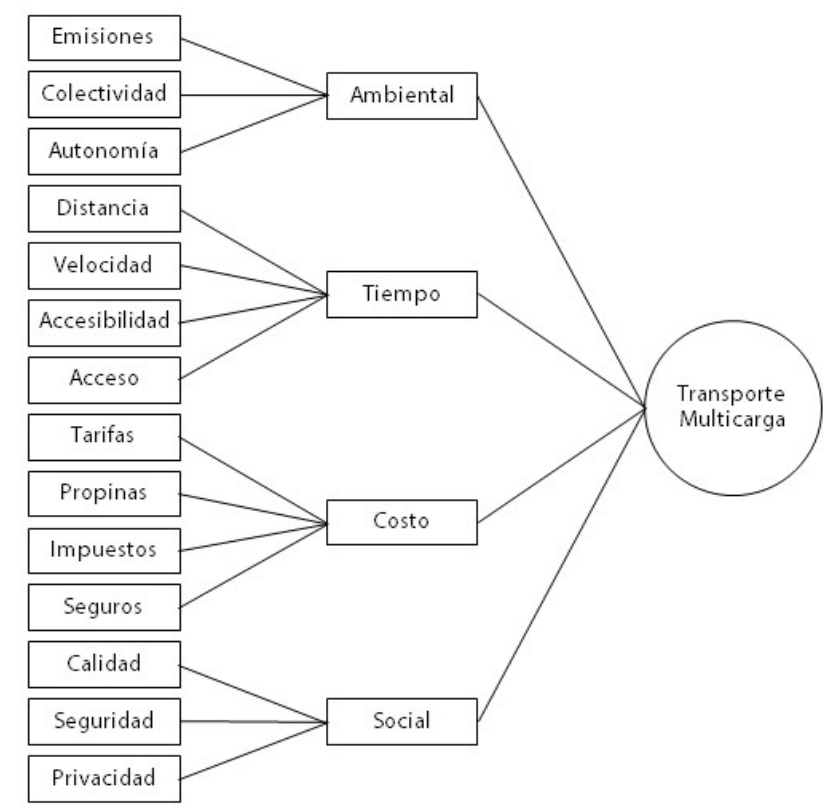

Fuente: Elaboración propia.

Además, la participación ciudadana y el cambio cultural son aspectos necesarios en este escenario, debido a que la presión social por la conciencia ambientalista es el disparador para la evolución del sistema del transporte. Por ejemplo, en Colombia se implementó una alianza públicoprivada en la cual se utilizó una App para mapear las rutas de autobús y definir así un uso más inteligente del transporte público por medio de los usuarios, quienes colaboran activamente y se ven beneficiados con menor tiempo de traslado, conocer la hora de llegada de una unidad a una determinada parada de autobús y mejorar su calidad de vida al pasar menos cantidad de horas al año en congestionamientos viales. Para lograrlo se utilizó la aplicación Moovit por parte de 1.5 millones colombianos (De Moya, 2014).

Los análisis estructural, de estrategia y morfológico permiten definir los principales factores y variables para identificar tendencias o puntos de ruptura (Godet et al, 2000) y en la aplicación teórica al caso de la empresa Uber con apoyo del comercio de servicios y literatura especializada se 
logran construir tres escenarios, de los cuales, dos se pueden clasificar de tendencia por la innovación en el servicio público y los esquemas robustos de colaboración privada; mientras que solo un escenario es de ruptura para concebir un esquema de servicio de transporte de carga múltiple.

\section{Discusión}

La evidencia documental encontrada permite afirmar que existe una diversificación del servicio brindado por la empresa Uber, sin embargo, en la práctica no se identifican procesos de pilotaje de convertirse en un servicio multicarga aprovechando los vehículos en su capacidad plena para distribución de personas y mercancías según los análisis efectuados.

En el análisis estructural aplicado se identifica el marco normativo costarricense, sus brechas y las disposiciones internas del país (leyes para servicio de taxi y porteo) que riñen con la habilitación de plataformas tecnológicas para la provisión de servicios privados de transporte de personas, porque variables como seguros, impuestos y tarifas están sin regulación. Asimismo, existe un marco internacional que podría contribuir a corregir estas distorsiones en el mercado.

En cuanto al análisis de estrategia, la empresa Uber ha evolucionado desde su creación con la diversificación de sus servicios, provocando cambios en la economía del transporte por medio de la economía compartida y asegurando la participación de más usuarios mediante modelos y esquemas organizacionales que apuntan a servicios culturales con divisiones específicas para grupos étnicos, lo cual fomenta el autoempleo, facilita la disposición de viajes compartidos y encadena la distribución de mercancías de consumo masivo y medicamentos.

Con el análisis morfológico, las cuestiones clave del futuro y los escenarios, se definen los factores ( $y$ variables) del servicio privado de personas y mercancías: tiempo (distancia, velocidad, accesibilidad, acceso), costo (tarifas, propinas, impuestos, seguros), social (calidad, seguridad, privacidad) y ambiental (emisiones, colectividad, autonomía).

Dichos factores y variables permiten establecer tres escenarios: servicio público innovador, transporte privado colaborativo y transporte multicarga. En los primeros dos escenarios se tienen procesos de innovación con base en las tendencias de incorporación de plataformas tecnológicas en el mercado y en la economía del transporte. En el tercer escenario se produce una ruptura con esas tendencias provocando una optimización en la utilización del vehículo como medio de transporte para cargas múltiples, es decir, mercancías y personas en un mismo viaje para una ruta óptima, combinando aspectos de tiempo, costos, sociales y ambientales. 
Al respecto, para estudios similares donde la información sobre los procesos de modernización del transporte público con una visión integral (legal, técnica, tecnológica, social, ambiental y humana, entre otras) sea escasa, se recomienda partir de un análisis teórico de escenarios, eliminando la generación de sesgos que limiten los análisis prospectivos para el mejoramiento de la toma de decisiones y la construcción de escenarios.

Desde la perspectiva de las ciencias de la administración, los factores definidos y sus variables identificadas pueden vislumbrarse como indicadores de desempeño que contribuyan a un mejor diseño de ecosistemas de transporte y logística. De esta forma, se estima que la Administración Pública podría contar con datos acerca de la calidad del servicio y la seguridad por vías terrestres, así como valorar la evolución de los servicios de transporte de personas y mercancías.

Si bien no se identificaron estudios similares, se considera que el presente estudio contribuye a la discusión científica en los campos del comercio de servicios y de la economía del transporte, así como en los estudios prospectivos teóricos y exploratorios de la economía compartida y de la conducta de los usuarios de plataformas tecnológicas, ya que, según lo estima el tercer escenario descrito, en un futuro los transportes serán multicarga: varios clientes con un mismo destino compartirán el vehículo con alguna mercancía de otra persona o empresa, logrando los objetivos de autoempleo, uso óptimo del uso del vehículo, reducción de costos, disminución de la huella de carbono, menores tiempos de desplazamiento $y$, en el caso de la compañía, aumento en las ganancias por las tarifas de interconexión entre transportistas y usuarios.

Finalmente, los factores, variables y escenarios definidos deben probarse mediante trabajo de campo para la recopilación de datos. Para análisis posteriores se propone un método mixto (cualitativo y cuantitativo) aplicando el método Delphi de Dalkey y Hermes, el análisis jerárquico de procesos de T. Saaty o el Triángulo Griego de M. Godet con el fin de responder a interrogantes como: ¿qué puede ocurrir?, ¿qué se puede hacer?, ¿qué va a hacerse? y ¿cómo se hará?

\section{Referencias Bibliográficas}

1. Alemi, F., Circella, G., Handy, S. \& Mokhtarian, P. (2018). What influences travelers to use Uber? Exploring the factors affecting the adoption of ondemand ride services in California. Travel Behaviour and Society, 13, 88-104. https://doi.org/10.1016/j.tbs.2018.06.002

2. Alley, J. K. (2016). The Impact of Uber Technologies on the New York City Transportation Industry. Finance Undergraduate Honors Theses. University of Arkansas. 31. https://scholarworks.uark.edu/finnuht/31 
3. Ávalos, M. y Sofía, P. (2015). Baby, you can ('t) drive my car. El caso de Über en Méjico. Economía Informa, 390m 104-112. https://doi.org/10.1016/S01850849(15)30007-4

4. Chuang, L. W., He, J. \& Chiu, S. P. (2018). Understanding User Participation in Sharing Economy Services. International Conference on Consumer Electronics-Taiwan (ICCE-TW). IEEE.

5. COMEX (2004). Tratado de Libre Comercio: República Dominicana - Centro América - Estados Unidos. Documento explicativo. Ministerio de Comercio Exterior. $118 \mathrm{pp}$.

6. Costa Rica. Asamblea Legislativa (1965). Ley No. 3503 reguladora del transporte remunerado de personas en vehículos automotores y sus reformas. Poder Legislativo.

7. Costa Rica. Asamblea Legislativa (1996). Ley No. 7593 de la Autoridad Reguladora de los Servicios Públicos y sus reformas. Poder Legislativo.

8. Costa Rica. Asamblea Legislativa (1999). Ley No. 7969 reguladora del servicio público de transporte remunerado de personas en vehículos en la modalidad de taxi y sus reformas. Poder Legislativo.

9. Costa Rica. Asamblea Legislativa (2011). Reforma de la Ley No. 3284, Código de Comercio, de 30 de abril de 1964, y de la Ley No. 7969, Ley reguladora del servicio público de transporte remunerado de personas en vehículos en la modalidad de taxi, de 22 de diciembre de 1999, No. 8955. Poder Legislativo.

10. Davidson, A. \& Fischer, L.A. (2015). (Re) Producing Transport Systems: Uber, Driverless Technology, and the Modern City. No. Conference: Transportation Research Board 94th Annual Meeting. https://trid.trb.org/view/1339208

11. De La Hoz Manotas, A. K.; Sepulveda-Ojeda, J. A. y Sarmiento-Polo, R. D. (2015). Prototipo móvil de realidad aumentada para sistema de transporte masivo en la ciudad de Barranquilla. Prospectiva, 13(2), 99-106. http://www. scielo.org.co/pdf/prosp/v13n2/v13n2a12.pdf

12. De Moya, M. E. (2014). Marco conceptual para el diseño de Modelos de Negocios para la prestación de Servicios Basados en Localización, en el ámbito colombiano (Tesis de maestría). Universidad Nacional de Colombia, Bogotá, Colombia.

13. De Rus, G., Campos, J. y Nombela, G. (2003). Principios de economía del transporte (1-20). En: DE RUS, Ginés et al. Economía del transporte. Barcelona.

14. Godet, M., Monti, R., Meunier, F. y Roubelat, F. (2000). La caja de herramientas de la prospectiva estratégica. Laboratoire d'Investigation Prospective et Stratégique, Prospektiker-Instituto Europeo de Prospectiva y Estrategia. 102 pp. http://es.laprospective.fr/dyn/espagnol/bo-lips-esp.pdf

15. Gómez, M., Galeano, C., Jaramillo, D. A. (2015). El estado del arte: una metodología de investigación. Revista Colombiana de Ciencias Sociales, 6(2), 423-442. https://doi.org/10.21501/22161201.1469

16. Granados, G. (2018). Esto dice ahora Hacienda sobre Uber y Airbnb. La Prensa Libre. http://www.laprensalibre.cr/Noticias/detalle/131274/esto-dice-ahorahacienda-sobre-uber-y-airbnb 
17. Laurell, C. \& Sandström, C. (2016). Analysing Uber in social media - disruptive technology or institutional disruption? International Journal of Innovation Management, 20(5), 1-19. https://doi.org/10.1142/S1363919616400132

18. Lloréns, F. J. (1995). Un análisis de la importancia relativa que tienen las dimensiones de la calidad de servicio en la percepción del cliente. Cuadernos de Ciencias Económicas y Empresariales, 29, 35-45. http://cuadernos.uma.es/ pdfs/pdf374.pdf

19. Miklos, T. (2002). Planeación prospectiva y estratégica. Ponencia en V Encuentro de Estudios Prospectivos. Guadalajara, Méjico. http://visitas.reduaz.mx/ coleccion_desarrollo_migracion/americalat/Americalat_capl_planeacion.pdf

20. Mora, E. J. y Arauz, J. A. (2016). Estudio prospectivo del sector de logística y transporte para el cambio de la matriz productiva de la Provincia del Carchi, marzo - julio 2015 (Tesis de maestría). Instituto de Investigación y Posgrado. Quito: UCE.

21. Mora-Mata, R.(2019).¿Podría una normativa regularla venta de medicamentos a través de Uber Eats? https://semanariouniversidad.com/bloque1/podriauna-normativa-regular-la-venta-de-medicamentos-a-traves-de-uber-eats/

22. Organización Mundial del Comercio. (1994). Acuerdo General sobre el Comercio de Servicios. Ginebra.

23. Powers, B. W., Rinefort, S., Jain, S. H. (2016). Nonemergency medical transportation: delivering care in the era of Lyft and Uber. JAMA, 316(9), 921922. https://doi.org/10.1001/jama.2016.9970

24. Rauch, D. E. \& Schleicher, D. (2015). Like Uber, but for local government law: the future of local regulation of the sharing economy. https://heinonline.org/ HOL/LandingPage?handle=hein.journals/ohslj76\&div=31\&id=\&page

25. Singh, M. (2019). Uber. Bloomberg Businessweek. https://www.bloomberg. com/features/companies-to-watch-2020/

26. Umaña, R. A. (2016). El Acuerdo General sobre el Comercio de Servicios (AGCS), características y asociación con salud (Tesis doctoral). Universidad de Alicante, España.

27. Wallsten, S. (2015). The competitive effects of the sharing economy: how is Uber changing taxis. Technology Policy Institute, 22. https:// techpolicyinstitute.org/wp-content/uploads/2015/06/the-competitiveeffects-of-the-2007713.pdf

28. Winter, K. (2020). Identifying user classes for shared and automated mobility services. European Transport Research Review, 12(1), 1-11. https://doi. org/10.1186/s12544-020-00420-y

Para citar este artículo:
Mora, E. O. (2020). Evolución del transporte de personas y mercancías. ¿Uber como medio de carga múltiple? Teuken Bidikay, 11(17), 197-217. doi: 10.33571/teuken.v11n17a11

\section{Ge: AMV}

